\title{
Generation of Monoclonal Antibodies against Human Prion Proteins in $\operatorname{PrP}^{\mathbf{0} / 0}$ Mice
}

\author{
Susanne Krasemann, ${ }^{*}$ Martin H. Groschup, ${ }^{\dagger}$ Silke Harmeyer, ${ }^{\dagger}$ \\ Gerhard Hunsmann, ${ }^{*}$ and Walter Bodemer* \\ *Department of Virology and Immunology, German Primate Center \\ (DPZ), Goettingen, Germany \\ ${ }^{\dagger}$ Federal Research Center for Virus Diseases of Animals, Tübingen, \\ Germany
}

\begin{abstract}
Background: Prion diseases belong to a group of neurodegenerative disorders affecting humans and animals. The human diseases include kuru, Creutzfeldt-Jakob disease (CJD), Gerstmann-Sträussler-Scheinker syndrome (GSS), and fatal familial insomnia (FFI). The pathogenic mechanisms of the prion diseases are not yet understood. Monoclonal antibodies provide valuable tools in the diagnosis, as well as in the basic research, of several diseases; however, monospecific antisera or monoclonal antibodies (mAbs) against human prion proteins were, until now, not available.

Materials and Methods: We have developed an immunization protocol based on nucleic acid injection into nontolerant $\mathrm{PrP}^{\mathrm{O} / 0}$ mice. DNA or RNA coding for different human prion proteins including the mutated sequences associated with CJD, GSS, and FFI were injected into muscle tissue. Mice were primarily inoculated with
\end{abstract}

DNA plasmids encoding the prion protein (PRNP) gene and boosted either with DNA, RNA, or recombinant Semliki Forest Virus particles expressing PRNP. Hybridomas were then prepared.

Results: Different mAbs against human prion proteins were obtained, and their binding behavior was analyzed by peptide enzyme-linked immunosorbent assay, Western blot, immunofluorescence, and immunoprecipitation. Their cross-reactivity with prion protein from other species was also determined. Our mAbs are directed against four different linear epitopes and may also recognize discontinuous regions of the native prion protein. Conclusions: These antibodies should allow us to address questions concerning the nature of the prion protein as well as the initiation and progression of prion diseases. Moreover, these mAbs can now be used for the diagnosis of prion diseases of humans and animals.

\section{INTRODUCTION}

Prions are pathogens, distinct from viroids and viruses, which cause diseases in animals and humans $(1,2)$. Prion diseases arise not only after transmission but also sporadically (3), and some of these neurodegenerative diseases are inherited (4). The most common animal diseases caused by prions are scrapie in sheep and bovine spongiform encephalopathy (BSE) in cattle. The human prion diseases include kuru, CreutzfeldtJakob disease (CJD), Gerstmann-Sträussler-

Address correspondence and reprint requests to: Walter Bodemer, Department of Virology and Immunology, German Primate Center, Kellnerweg 4, 37077 Goettingen, Germany.
Scheinker syndrome (GSS) and fatal familial insomnia (FFI). GSS, FFI, and about $10 \%$ of CJD cases represent dominantly inherited disorders with defined mutations within the PRNP gene located on chromosome 20p (5). In contrast, about $90 \%$ of CJD cases occur sporadically. CJD has been repeatedly transmitted iatrogenically (6). The human prion diseases can be transmitted experimentally to laboratory animals, with induction of disease after long incubation periods (7).

During the progression of prion disease, protein aggregates accumulate in the brain of the affected organisms (8). These plaques are largely, if not entirely, composed of an abnormal, protease-resistant isoform of the host-encoded prion 
protein (9). The central role of this disease-associated prion protein isoform in transmission and pathogenesis of prion diseases has been confirmed by independent experimental approaches (10). Unexpectedly, prion protein-deficient mice $\left(\mathrm{PrP}^{0 / 0}\right.$ mice) are viable and do not show obvious changes in development, behavior, or reproduction (11). Thus, the normal function of the protein still remains to be elucidated. However, the $\mathrm{PrP}^{0 / 0}$ mice are resistant to transmission of prion diseases (12).

Monoclonal antibodies (mAbs) against prion proteins and their pathogenic isoforms could be valuable tools to discriminate between individual forms of the protein and to determine their topology. Such antibodies could also help elucidate the structure and function of the cellular prion protein. To date, only a limited number of monospecific antisera and mAbs against prion proteins are available (13-16). None of these is human specific, and they do not differentiate between the normal and the disease-associated isoforms. This lack of appropriate reagents is partly due to the immune tolerance of laboratory animals against their own prion proteins and to the genetically related human prion proteins. In addition, routine immunizations with well-defined peptides or with prion protein preparations from brain tissue may not mimic the intracellular pathways necessary for correct and efficient processing and presentation of immunogenic parts of these proteins. Furthermore, the conformation of the protein isoforms may not have been preserved during purification procedures. Thus, the quality of the antibodies induced may have been impaired.

It has already been shown that $\mathrm{PrP}^{0 / 0}$ mice synthesize polyclonal antibodies against prion proteins after immunization with purified protein (17). However, mAbs have not yet been generated. Instead of immunizing mice with prion proteins or peptides, we used a nucleic acid-mediated immunization protocol with vector constructs comprising the open reading frame (ORF) of different human prion genes. We showed that immune sera of mice bind to prion proteins in a peptide-specific enzyme-linked immunosorbent assay (ELISA), Western blot, radioimmunoprecipitation and immunofluorescence (18).

In the experiments described below, we boosted the immune response of $\mathrm{PrP}^{0 / 0}$ mice by three different techniques. Hybridoma lines were established with spleen cells of these mice and screened for secretion of mAbs against prion pro- tein. Using the technique described, $\operatorname{PrP}^{0 / 0}$ mice were used successfully to generate mAbs against human prion proteins. These mAbs are not only directed against four distinct linear epitopes of the protein. They also exhibit different binding characteristics when analyzed in peptide ELISA, Western blot, immunofluorescence, and radioimmunoprecipitation. Therefore, these antibodies may also recognize and bind to discontinuous epitopes of the native prion protein.

\section{MATERIALS AND METHODS}

\section{Materials}

All chemicals were of the highest purity commercially available.

\section{Animals}

Prion protein-deficient $\mathrm{PrP}^{0 / 0}$ mice kindly provided by Charles Weissmann were used for DNA-mediated immunization (11).

\section{Vector Construction}

Construction of vectors for DNA-mediated immunization has been described elsewhere (18). In brief, the entire ORF of the PRNP gene of a healthy person or of individual patients with prion diseases was amplified by polymerase chain reaction (PCR), cloned into the pCDNA3vector (Invitrogen, NV Leek, The Netherlands), and confirmed by sequencing. Three different genotypes were cloned by this method: the cellular wild-type gene, a GSS-associated prion gene with an P102L mutation, and a novel insertion mutant comprising 14-octapeptide repeats within the repetitive region of the prion gene (19). Five different disease-associated mutations within the prion gene, which could not be amplified from patient's DNA, were introduced by in vitro mutagenesis into the cellular ORF. These mutations are associated with GSS (ala 117val; phe198ser), CJD (aspl78asn in combination with 129val; glu200lys), and FFI (aspl78asn in combination with 129met).

\section{Construction of rSFV Vectors}

The ORF of different prion genes were subcloned into the vectors $\mathrm{pBK}-\mathrm{CMV}$ (Stratagene, La Jolla, CA, U.S.A.) and thereafter into $\mathrm{pBS}^{+}$(Stratagene) to provide compatible BamHI restriction 
sites for subsequent cloning into the vector pSFVl, kindly provided by P. Liljeström (20).

\section{RNA Syntheses in Vitro}

pSFV-PrP DNA plasmids for in vitro transcription were linearized with SpeI. RNA was synthesized at $37^{\circ} \mathrm{C}$ for $\mathrm{l} \mathrm{hr}$ in $50 \mu \mathrm{l}$ containing $5 \mu \mathrm{g}$ of linearized DNA, $40 \mathrm{mM}$ HEPES-KOH (pH 7.4), 6 $\mathrm{mM} \mathrm{MgCl} 2,2 \mathrm{mM}$ spermidine- $\mathrm{HCl}, 5 \mathrm{mM}$ dithiothreitol (DTT), $100 \mu \mathrm{g} / \mathrm{ml}$ of nuclease-free bovine serum albumin (BSA), $1 \mathrm{mM}$ each of ATP, CTP, and UTP, $500 \mu \mathrm{M}$ GTP, $1 \mathrm{mM}$ $7 \mathrm{G}\left(5^{\prime}\right) \mathrm{ppp}\left(5^{\prime}\right) \mathrm{G}, 1$ unit/ $\mu \mathrm{l}$ of RNAasin, and 500 units/ml of SP6 RNA polymerase. RNA was either directly used for transfection by electroporation of cells or purified with a commercially available kit (Diagen, Düsseldorf, Germany) to prepare highly concentrated RNA for RNA-mediated immunization of mice.

\section{Electroporation}

Baby hamster kidney (BHK) cells were trypsinized, washed once with cell culture medium and once with phosphate-buffered saline (PBS) (without $\mathrm{MgCl}_{2}$ and $\mathrm{CaCl}_{2}$ ), and finally resuspended in PBS, to give $10^{7}$ cells $/ \mathrm{ml}$. Cells in a volume of 0.8 $\mathrm{ml}$ were transferred to a $0.2-\mathrm{cm}$ cuvette (Biorad, Richmond, CA, U.S.A.), $50 \mu \mathrm{l}$ of unpurified in vitro transcribed RNA solution was added, and electroporation was carried out at room temperature by two consecutive pulses at $1.5 \mathrm{kV} / 25 \mu \mathrm{F}$ using a BioRad Gene Pulser apparatus with its pulse controller unit set at maximum resistance. Before the cells were transferred into tissue culture plates, they were diluted at least 1:20 in complete BHK-cell medium (BHK21 [Glasgow MEM], $5 \%$ fetal calf serum [FCS, v/v], 10\% tryptose-phosphate broth [v/v], 20 mM HEPES, 2 mM glutamin).

\section{Preparation of Recombinant Semliki Forest Virus Particles}

RNA transcribed in vitro from pSFV-PrP DNA was mixed with helper 2 RNA at a ratio of $1: 1$ and cotransfected by electroporation into BHK cells as described above. Cells were grown for 24 hr before the culture medium (complete BHKmedium) was collected. This supernatant contains inactive virus. After activation of virus particles by adding chymotrypsin to a final concentration of $0.5 \mathrm{mg} / \mathrm{ml}$ for $30 \mathrm{~min}$, the reaction was stopped with 0.5 volumes of aprotinin concentrated to $2 \mathrm{mg} / \mathrm{ml}$ (21). The solution, which contained approximately $10^{5}-10^{6}$ infectious particles per milliliter, was used immediately to inoculate animals.

\section{Immunization Protocol}

DNA or RNA was injected into a regenerating muscle (22). Five days prior to nucleic acid immunization, mouse muscle tissue was pretreated with cardiotoxin (10 $\mu \mathrm{M}$ in PBS) purified from the venom of the Naja nigricollis snake (Latoxan, Rosans, France). The effects of cardiotoxin have been described in detail previously (23). $\mathrm{PrP}^{0 / 0}$ mice each received bilateral injections with 100 $\mu \mathrm{l}$ of the cardiotoxin solution into the tibialis anterior muscle with a 27-gauge needle. Five days later, either $50 \mu \mathrm{g}$ plasmid DNA or $5 \mu \mathrm{g}$ RNA coding for human PrP was injected into each muscle. The DNA was dissolved in PBS at a concentration of $1 \mu \mathrm{g} / \mu \mathrm{l}$ DNA in PBS. The RNA concentration was $100 \mu \mathrm{g} / \mathrm{ml}$ in PBS. Splenocytes of immunized mice were prepared and fused 7 days after DNA or 3 days after RNA injection. Recombinant SFV prion particles were activated with chymotrypsin. Particles $\left(10^{5}-10^{6}\right)$ were injected once intraperitoneally into $\operatorname{Pr}^{0 / 0}$ mice. Preparation of splenocytes and fusions were performed 2 days after infection with virus particles.

\section{Fusion Protocol}

The fusion of permanently growing P3X63 Ag8.653-mouse myeloma cells (ATCC CRL 1580) (24) with splenocytes from immunized $\mathrm{PrP}^{0 / 0}$ mice was performed as described by Peters and Baumgarten (25).

\section{ELISA}

The ELISA protocol has been described elsewhere (18). In brief, ELISA plates were coated with 100 ng per well with 1 of 16 different overlapping peptides. These peptides were derived from the sequence of the human prion protein and comprised the entire protein. In addition, a peptide containing the FFI-associated mutation was also tested in the ELISA assay. The reaction against the mutated amino acid sequence was compared with that of the cellular protein. For the ELISA assay, mouse sera were diluted 1:200 in PBS containing 3\% FCS, whereas hybridoma supernatant was diluted $1: 10$. Antibodies binding 
to the peptides were detected with a horseradish peroxidase-OPD system.

\section{Western Blot}

For Western blot analyses, prion proteins isolated from brain tissues of different species were used. Proteins were extracted from tissues according to Pan et al. (26). Proteins were separated on a $13 \%$ polyacrylamide gel. For immunoblotting, proteins were electrophoretically transferred onto nylon membrane (Boehringer, Mannheim, Germany). The detection of prion proteins by the antibodies were carried out according to Pan et al. (26). Recombinant human prion proteins were obtained from lysed BHK cells after electroporation with in vitro transcribed SFV-RNA containing different PrP ORFs. Twenty-four hours later, BHK cells were harvested with a cell scraper, washed twice in PBS, and resuspended in an appropriate volume of bidistilled water. Cells were repeatedly frozen and thawed. Thereafter, one volume of reducing sample buffer was added. Proteins were separated electrophoretically on a $12.5 \%$ polyacrylamide gel, transferred onto membranes and detected as described above (26).

\section{Immunofluorescence}

BHK cells were electroporated with in vitro transcribed SFV-PrP RNA. They were subsequently grown for $20 \mathrm{hr}$ and washed once with PBS before fixation with methanol and acetone (3:1 $\mathrm{v} / \mathrm{v}$ ) at room temperature for $2 \mathrm{~min}$. Cells were washed twice with PBS supplemented with $3 \%$ FCS and incubated with serum of immunized mice or hybridoma supernatant for at least $1 \mathrm{hr}$ at $37^{\circ} \mathrm{C}$. Sera were prediluted 1:200 and hybridoma supernatants 1:4 with 3\% FCS in PBS. Subsequently, cells were washed three times and a FITC-coupled sheep anti-mouse antibody was added at a dilution of $1: 200$ in 3\% FCS in PBS. Cells were incubated for $30 \mathrm{~min}$ at $37^{\circ} \mathrm{C}$, washed three times and analyzed with a ultraviolet light immunofluorescence microscope.

\section{RESULTS}

Using $\mathrm{PrP}^{0 / 0}$ mice, we induced both polyclonal antisera and a panel of mAbs against human prion proteins. To circumvent the preparation of potentially pathogenic prion proteins from human brain tissues, we conducted immunizations with nucleic acids. Distinct genotypes of the human prion ORFs were cloned into an appropriate vector under the control of the cytomegalovirus (CMV) promoter. The PRNP genotypes consisted either of the normal wild-type sequence or contained distinct mutations associated with the human prion diseases Creutzfeldt-Jakob disease (CJD), Gerstmann-Sträussler-Scheinker syndrome (GSS), and fatal familial insomnia (FFI).

\section{Immunization of Nontolerant $\operatorname{PrP}^{0 / 0}$ Mice}

$\mathrm{PrP}^{0 / 0}$ mice were immunized once with $100 \mu \mathrm{g}$ purified DNA of the vector constructs into the regenerating muscle tissue pretreated with cardiotoxin. As a control, muscle tissue of the $\mathrm{PrP}^{+/+} \mathrm{BALB} / \mathrm{c}$ mice was also treated with cardiotoxin and received the same amount of purified DNA as the $\mathrm{PrP}^{0 / 0}$ mice. Nine weeks after the first DNA injection, immune sera of mice were tested for prion-specific antibodies. In the peptide ELISA, antibodies against human PrP were not detected in sera from immunized $\operatorname{PrP}^{+1+}$ $\mathrm{BALB} / \mathrm{c}$ mice. Sera from these mice also failed to bind to human prion protein in an immunofluorescence assay. In striking contrast, all immunized $\operatorname{Pr}^{0 / 0}$ mice developed a polyclonal antibody response against prion proteins. The most efficient immune response against prion proteins could be repeatedly induced by DNA-mediated immunization of $\mathrm{PrP}^{0 / 0}$ mice with an ORF sequence containing the FFI-associated mutation at amino acid 178. Therefore, the mice immunized with this construct were chosen for further experiments. To enhance the induction of polyclonal antibodies in these mice and the number of blastocytes in the spleen, booster immunizations were performed by three different strategies 10 weeks after the first DNA injection. Mice were boosted by injecting $100 \mu \mathrm{g}$ of plasmid DNA into the regenerating muscle. Alternatively, mice received $5 \mu \mathrm{g}$ RNA injected into the regenerating muscle tissue. RNA was synthesized in vitro from a Semliki Forest Virus (SFV) repliconbased DNA plasmid containing the corresponding human FFI-associated PrP ORF. In a third approach, $\mathrm{PrP}^{\mathrm{O} / 0}$ mice were boosted intraperitoneally with approximately $10^{5}-10^{6}$ infectious recombinant SFV particles encoding the human FFI-associated prion protein. 


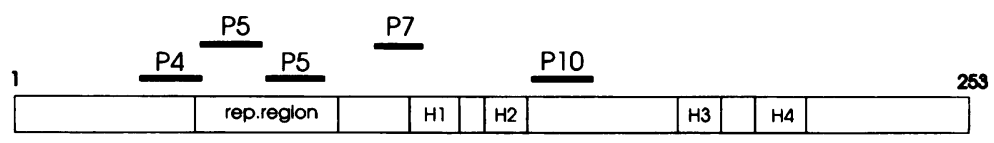

$\begin{array}{lll}\text { P4 } & \text { RYPGQGSPGGNRYPPQG } & \text { mab 14D3 } \\ \text { P5 } & \text { GGGWGQPHGGGWGQPH } & \text { mab } 4 \mathrm{~F} 2 \\ \text { P7 } & \text { THSQWNKPSKPKTNMK } & \text { mab } 8 G 8 \\ \text { P10 } & \text { GSDYEDRYYRENMHRYPNQ } & \text { mab } 12 \mathrm{~F} 10\end{array}$

FIG. 1. Mapping human prion protein epitopes with monoclonal antibodies in a peptide ELISA assay

The ELISA assays were performed using 16 partially overlapping peptides derived from the sequence of the human prion protein. The entire prion protein (amino acids 1-253), its repetitive region (rep. region), and the four putative helix regions (H1-H4) are shown. The monoclonal antibodies used, the epitope-carrying peptides, their intramolecular localization, and primary sequences are given in the lower part of the figure.

\section{Splenocyte Fusion}

Spleen cells of each immunization group were fused to permanently growing mouse myeloma cells. The number of blastocytes in the spleen of these mice differed extensively depending on the immunization protocol. The numbers ranged from 0.9 to $1.8 \times 10^{7}$ after immunization with nucleic acid, regardless of whether DNA or RNA was used for the booster immunization. Blastocyte numbers were significantly higher, up to 5.1 and $6.7 \times 10^{7}$, after boosting with recombinant SFV particles.

To evaluate whether hybridomas were capable of synthesizing mAbs against linear and putative conformational epitopes of the prion protein, supernatants were screened by peptide ELISA, Western blot, immunofluorescence, and radioimmunoprecipitation.

\section{Epitope-Specific Binding of mAbs in a Peptide ELISA}

To determine the specificity of the mAbs, peptide ELISAs were performed with a panel of overlapping 16-mer peptides, representing the complete sequence of the human prion protein (Fig. 1). Two mAbs, 14D3 and 4F2, bound to peptides 4 and 5 , respectively, which represent a region upstream of or within the repetitive domain. Two additional mAbs were obtained against linear epitopes. mAb $8 G 8$ is directed against a region upstream of the proposed first helix region (H1). The other monoclonal antibody, 12F10, binds to a region downstream of the neurotoxic domain adjacent to the helix region 2 (Fig. 1). None of the mAbs showed reactivity against the peptide containing the FFI-associated mutation or the corresponding wild-type peptide.

\section{Binding of mAbs by Western Blot Analysis}

The results of the peptide ELISA were confirmed by Western blot analyses. Each of the four mAbs was capable of detecting prion proteins purified from human brain homogenates. mAb $4 \mathrm{~F} 2$, directed against the repetitive region, gave the strongest signal. Faint signals were obtained with mAbs 14D3, 8G8, and 12F10. These antibodies also detected the normal human prion proteins synthesized in BHK cells after electroporation with recombinant SFV RNA containing different PrP ORFs.

In Western blots containing $\operatorname{PrP}^{c}$ prion protein preparations from different species, two of these antibodies, 14D3 and 4F2, showed a broad and strong reactivity against most species tested, including the ovine, bovine, mouse, hamster, and human prion protein (Fig. 2a). These antibodies, recognizing peptide 4 or 5 only, failed to detect the prion protein of guinea pigs. mAb 12F10, which reacts with peptide 10 , showed only faint signals in these analyses. In contrast, the monoclonal antibody 8G8, directed against peptide 7 , reacted strongly with the bovine prion protein (Fig. 2b). It also detected the ovine and the human proteins but failed to bind to any other mammalian cellular prion proteins tested so far (Fig. 2b).

We demonstrated that four mAbs bound to linear epitopes of the prion protein in a peptide ELISA. These mAbs also reacted against the denatured cellular prion proteins on Western blots. To evaluate whether our mAbs could recognize discontinuous epitopes on the native prion protein, we performed immunofluorescence and radio immunoprecipitation analyses.

\section{Reactivity of mAbs with Transfected BHK Cells in the Immunofluorescence}

Immunofluorescence assays were performed with BHK target cells expressing human prion proteins. In vitro synthesized SFV-RNA, containing either the normal prion ORF or the mutated form associated with human FFI, were trans- 

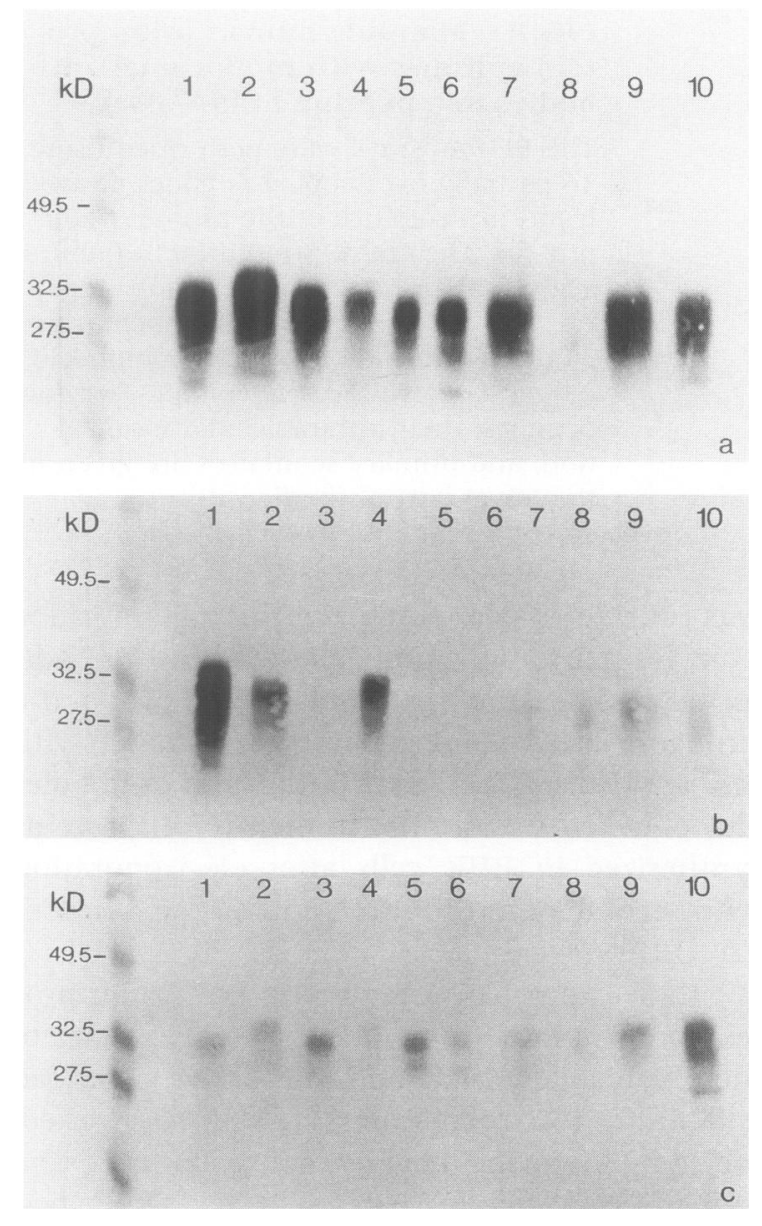

FIG. 2. Recognition of species-specific prion proteins by monoclonal antibodies in Western blots

The binding of the three monoclonal antibodies 4F2 (a), 8G8 (b), and 7B4 (c) to cellular prion proteins from sheep (Lane 1), cattle (2), pig (3), man (4), dog (5), rabbit (6), rat (7), guinea pig (8), hamster (9) and mouse $(10)$ was tested. Signals obtained after staining represent naturally occurring, processed prion protein molecules of apparent molecular sizes ranging from 27.5 to $35 \mathrm{kD}$. Left lane contains molecular weight markers.

ferred into these cells by electroporation. All mAbs that were positive in the peptide ELISA recognized the target protein in cells expressing the cellular as well as the FFI-associated human prion protein (Fig. 3b). As expected, there was no immunofluorescence with normal nonelectroporated BHK cells or with BHK cells transfected with RNA from the pSFVI cloning plasmid only. Preimmune sera of immunized mice, as well as cell culture supernatant from Ag8 mouse myeloma cells, also failed to bind to the human prion protein expressed in BHK cells (Fig. 3a).
mAb 8 G8 stained the prion protein expressing cells with a brilliant cytoplasmic fluorescence. However, the number of positive cells was small when compared with the number of stained cells detected with the other three mAbs or with polyclonal antisera. The transfection efficiencies were comparable in these assays, since one transfection was plated in several wells and prion protein expression was determined with different mAbs in parallel.

Moreover, we were able to establish three hybridoma lines, 7B4, 11C6, and 12E8, that secreted antibodies reacting with the prion protein as determined by immunofluorescence but did not bind to any peptide in the ELISA. mAb 7B4 was also tested in RIPA with ${ }^{35} \mathrm{~S}$-labeled recombinant prion protein and precipitated the target prion protein specifically (data not shown). In contrast, it failed to react in the Western blot with cellular $\operatorname{PrP}^{\mathrm{c}}$ from human brain tissue and recombinant cellular PrP. mAb 7B4 was also tested in Western blot with prion protein preparations from different species. However, very faint prion protein-specific signals could be obtained in this blot for all species tested. Surprisingly, only the human and the guinea pig prion proteins were not recognized in this assay, but the mouse $\operatorname{PrP}^{\mathrm{c}}$ was recognized quite well (Fig. 2c).

Our results have clearly shown that $\operatorname{PrP}^{0 / 0}$ mice can elicit mAbs against human prion proteins. These mAbs are directed against four different linear epitopes, as well as discontinuous epitopes, on the native prion protein. All mAbs described contain heavy chains from the IgG2a or $2 \mathrm{~b}$ subtype. Table 1 summarizes the binding specificities of some relevant mAbs.

\section{DISCUSSION}

To prepare mAbs against human prion proteins (PrP), we inoculated PrP-deficient mice with DNA comprising the open reading frame (ORF) of human prion protein genes (PRNP). These mice produced polyclonal antibodies and hybridomas secreting mAbs were established. This result may be attributed primarily to the immunological responsiveness of $\mathrm{PrP}^{\mathrm{O} / 0}$ mice against any prion protein. Although the tolerance of these null mice has been known for quite some time, we are not aware of any report of their use to generate mAbs raised specifically against human prion proteins. Perhaps our immunization strat- 

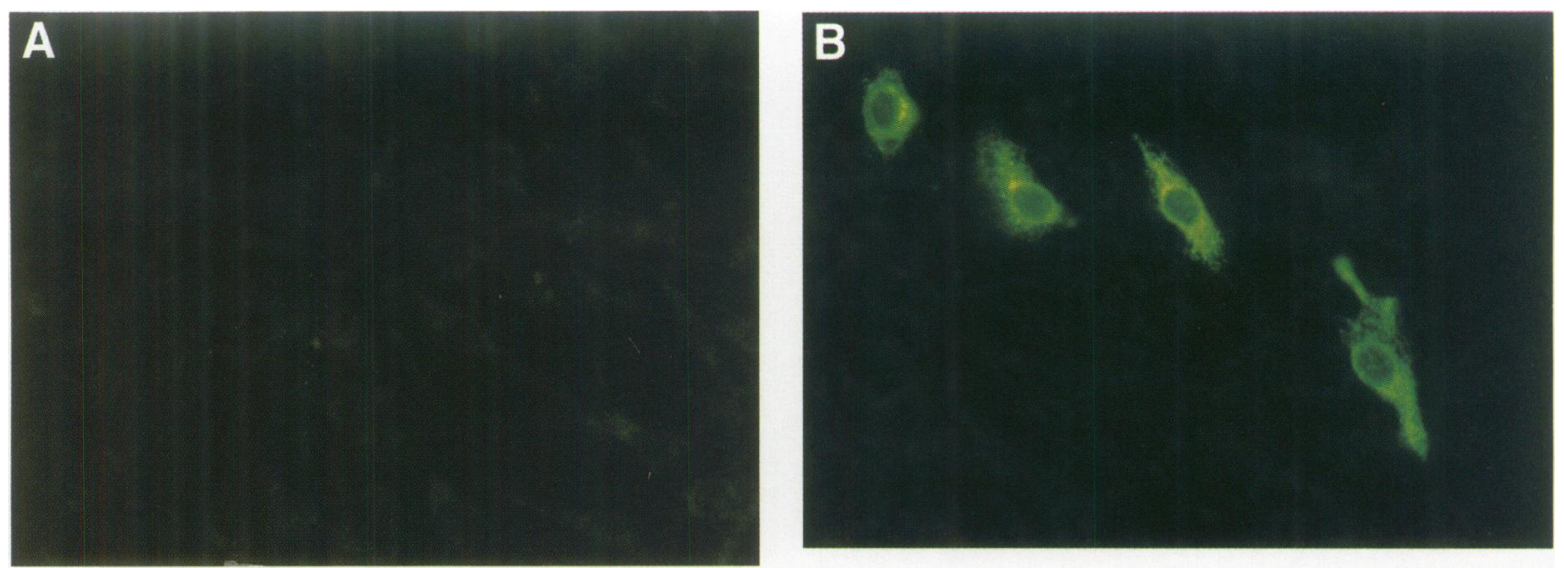

FIG. 3. Detection of human prion proteins by immunofluorescence with mAb $4 F 2$

BHK cells were transfected with chimeric SFV RNA containing the open reading frame (ORF) sequence of the cellular prion protein. At $24 \mathrm{hr}$ after electroporation, cells were fixed with methanol-acetone and stained with the monoclonal antibody. Culture supernatant from Ag8-myeloma cells did not react with BHK cells electroporated with recombinant SFV-RNA containing the cellular prion ORF sequence (a). In contrast, brilliant staining of BHKcells expressing recombinant prion proteins could be detected after incubation with mAb 4F2 (b).

egy contributed considerably to our success in obtaining mAbs.

With our immunization protocol, we obtained a total of 274 hybridomas. When tested in peptide ELISA and immunofluorescence assays, we found 46 hybridomas secreting mAbs against human prion proteins. This frequency of prion protein-specific hybridomas is significantly higher than that reported by Kascsak and colleagues (13). They screened more than 400 hybridomas and found only three secreting mAbs. Only one of these three had been

TABLE 1. Binding characteristics of mAbs directed against human prion proteins

\begin{tabular}{|c|c|c|c|c|c|}
\hline \multirow[b]{2}{*}{$\mathbf{m A b}$} & \multirow[b]{2}{*}{ Peptide ELISA } & \multicolumn{2}{|c|}{ WB } & \multirow[b]{2}{*}{ IF } & \multirow[b]{2}{*}{ RIPA } \\
\hline & & $\operatorname{PrP}^{\mathbf{c}}$ & recPrPc & & \\
\hline $14 \mathrm{D} 3$ & $\mathrm{p} 4$ & $+1-$ & $+1-$ & ++ & nd \\
\hline $4 \mathrm{~F} 2$ & p5 & +++ & +++ & +++ & ++ \\
\hline $13 F 10$ & p5 & ++ & ++ & +++ & nd \\
\hline $8 \mathrm{G} 8$ & p7 & + & + & $+^{a}$ & ++ \\
\hline $11 \mathrm{~B} 9$ & plo & ++ & + & ++ & nd \\
\hline $12 \mathrm{Fl} 0$ & plo & ++ & + & ++ & nd \\
\hline $7 \mathrm{~B} 4$ & - & - & - & + & ++ \\
\hline $11 \mathrm{C} 6$ & - & - & - & ++ & nd \\
\hline $12 \mathrm{E} 8$ & - & - & - & ++ & nd \\
\hline
\end{tabular}

The binding specificity of some of the mAbs described in this paper are summarized. The entire cellular human prion protein or parts thereof were used as a target for antibody binding. Overlapping peptides were taken to characterize the epitopes relevant for $\mathrm{mAb}$ recognition in a peptide ELISA. mAbs were directed against peptides 4, 5, 7, and 10 (p4, p5, p7, and p10). Western blots (WB) were performed using $\operatorname{PrP}^{\mathrm{c}}$ from human brain tissue $\left(\operatorname{PrP}^{\mathrm{c}}\right)$ or recombinant human prion protein from overexpressing BHK cells $\left(\mathrm{recPrP}^{\mathrm{c}}\right)$. These prion protein-expressing cells also served as a target for immunofluorescence assays (IF) or as source of metabolically labeled prion protein for radioimmunoprecipitation (RIPA). nd, not done.

${ }^{a} \mathrm{mAb} 8 \mathrm{G} 8$ showed a distinct binding pattern by exclusively staining a subset of prion protein expressing cells, as described in Results. 
characterized. This monoclonal antibody, 3F4, recognizes both human and hamster prion protein. It is directed against a region within the first putative helix, spanning the four amino acids $109-112$ (met, lys, his, met). In contrast, our mAbs recognized epitopes distributed over the entire prion protein molecule. Eleven were directed against peptide 4, 21 against peptide 5, only 1 against peptide 7 , and 7 against peptide 10 in the peptide ELISA assay. Six out of the 46 mAbs scored positive in immunofluorescence assays but not in peptide ELISA or Western blot analyses with human cellular PrP. Perhaps, these mAbs reacted with discontinuous epitopes which are still preserved after treatment with methanol and acetone. As a result, these mAbs may detect discontinuous, conformational epitopes rather than fully denatured, linear epitopes. Such mAbs would be most appropriate to investigate in vitro induced conformational changes of PrP. To examine if our mAbs recognize these discontinuous epitopes, we have performed radioimmunoprecipitation assays. The mAb 7B4 specifically precipitates recombinant prion protein encoded by the FFI-genotype sequence. Using immunofluorescence and radioimmunoprecipitation assays, one might detect mAbs discriminating between conformational changes within one prion protein molecule or between the nonpathogenic and pathogenic prion isoforms.

In our previous study, DNA-mediated vaccination by itself resulted in a strong polyclonal $\mathrm{B}$ cell response. However, to enhance the number of blastocytes for fusion with myeloma cells, we had to infect DNA-immunized animals with recombinant live-attenuated Semliki-Forest virus (SFV). Recombinant SFV helper 2 particles were prepared, each carrying chimeric RNA coding for nonstructural proteins of SFV and single different ORFs of the human PRNP. The systemic infection with SFV in combination with the initial local deposition of ORF sequences may have accounted for the larger number of blastocytes obtained. In contrast, boosting the immune response by a second inoculation of either DNA or RNA into muscle tissue was inefficient compared with the SFV infection. No hybridomas were obtained after the booster immunization with only DNA or RNA. We learned that DNA-mediated vaccination is not sufficiently efficient for generating blastocytes for hybridomas secreting mAbs. This may be due to a low level expression of exogenous plasmid DNA delivered into muscle tissue. To circumvent this problem, mice were infected with rSFV. The SFV-infected mouse cells may have synthesized the heterologous protein abundantly. In addition, the systemic infection with rSFV may involve, in particular, spleen cells and thereby induce a strong B cell response.

After delivery of nucleic acids expressing prion sequences or of the prion ORF by rSFV particles in the cells of mice, protein molecules are presumably synthesized in an authentic conformation. Thus, processing and presentation of the in vivo expressed foreign prion proteins to the immune system should be followed by a specific immune response. This may support a correct processing and presentation of all putative immunogenic regions by antigen-presenting cells. Several groups have demonstrated that immunization of mice with DNA encoding viral antigens leads to protective immunity against a subsequent virus challenge $(27,28)$. These data strongly recommended the use of this immunization strategy, since the immune response induced is equal if not superior to immunizations with protein or peptide preparations. Using this technique, we have avoided the prion protein preparation that could disturb the native conformation. Since mice naturally synthesize endogenous prion proteins, $\mathrm{PrP}^{0 / 0}$ mice should also possess the mechanisms to transcribe, translate, and process our exogenously introduced prion genes.

The mAbs obtained were also analyzed for interspecies cross-reaction. The cellular prion proteins were prepared from brain tissue of different species and analyzed in Western blot assays. A broad, positive reactivity with all species tested, except guinea pig, was obtained with all mAbs directed against peptide 5 . Since this peptide region is degraded by protease digestion in the disease-associated prion rods, we expect that these mAbs against peptide 5 do not recognize the $\mathrm{N}$-terminal truncated prion rods. The mAbs against peptide 10 showed only faint signals on Western blots. Interestingly, mAb 8G8 directed against peptide 7 reacted with three species only: cattle, sheep, and humans. A closer look at the corresponding peptide sequences does not explain this restricted interspecies binding specificity, since the bovine peptide sequence does not differ from that of mouse and hamster within the corresponding region. Of course, we cannot exclude that there may be amino acid polymorphisms between the published sequences and the PrP protein sequences we used for the Western blot analyses. Moreover, mAb 8G8 behaved quite differently in the immunofluorescence assays. In contrast to all other mAbs, mAb $8 \mathrm{G} 8$ reacted only with a subpopulation of prion pro- 
tein-expressing cells, although the transfection efficiencies were the same for all mAbs tested. One may speculate that some of the cells express PrP in a distinct conformation; this, of course, needs to be proven. Another reason for the unexpected binding patterns in Western blot and immunofluorescence assays could involve the cell-specific glycosylation of the respective target PrP proteins, especially if BHK cells express the target proteins for immunofluorescence assays.

Although mAb 7B4 does not react with linear epitopes in the peptide ELISA and Western blot assays with human prion protein, faint signals against the prion proteins of all species except humans and guinea pig could be observed.

After DNA-mediated immunization, PrP-deficient mice mount a strong humoral immune response against normal human prion protein and, although there is no conclusive evidence, to its respective pathogenic isoform. A combination of DNA-mediated immunization and boosting with rSFV-expressing individual ORFs of PRNP results in a large number of blastocytes, a prerequisite to establishing hybridomas. A panel of mAbs was obtained. Most of them reacted with linear epitopes, but some of them seem to recognize discontinuous epitopes, according to immunofluorescence and RIPA analyses. We expect these mAbs to become valuable tools for diagnostics and for basic research into the molecular properties of prion proteins.

\section{ACKNOWLEDGMENTS}

We thank Professor Charles Weissmann (Zurich, Switzerland) for providing the $\mathrm{PrP}^{0 / 0}$ mice. Technical help in establishing the hybridoma fusions was provided by H. Peters and U. Friedrichs (Göttingen, Germany). P. Liljeström and P. Berglund (Stockholm, Sweden) kindly provided the pSFVl and helper 2 plasmids. The skillful technical assistance of T. Jürgens is gratefully acknowledged. This project was supported by Grant 01KI9463 from the German BMBF.

\section{REFERENCES}

1. Prusiner SB. (1982) Novel proteinacious infectious particles cause scrapie. Science 216: 136-144.

2. Prusiner SB. (1991) Molecular biology of prion diseases. Science 252: 1515-1522.
3. Prusiner SB, and Hsiao KK. (1994) Human prion diseases. Ann. Neurol. 35: 385-395.

4. Brown P, Goldfarb LG, Gajdusek CD. (1991) The new biology of spongiform encephalopathy: Infectious amyloidoses with a genetic twist. Lancet 337: 1019-1022.

5. Robakis NK, Devine-Gage EA, Jenkins EC, et al. (1986) Localization of a human gene homologous to the PrP gene on the $p$ arm of chromosome 20 and detection of PrP-related antigens in normal human brains. Biochem. Biophys. Res. Commun. 140: 758-765.

6. Brown P, Cervenakowa L, Goldfarb LG, et al. (1994) Iatrogenic Creutzfeldt-Jakob disease: An example of the interplay between ancient genes and modern medicine. Neurology 44: 291-293.

7. Gibbs Jr CJ, Gajdusek DC, Asher DM, et al. (1968) Creutzfeldt-Jakob disease (spongiform encephalopathy): Transmission to the chimpanzee. Science 161: 388-389.

8. Brown $\mathrm{P}$, Coker Vann M, Pomeroy $\mathrm{K}$, et al. (1986) Diagnosis of Creutzfeldt-Jakob disease by Western blot identification of marker protein in human brain tissue. N. Engl. J. Med. 314: 547-551.

9. Meyer RK, McKinley MP, Bowman KA, Braunfeld MB, Barry RA, Prusiner SB. (1986) Seperation and properties of cellular and scrapie prion proteins. Proc. Natl. Acad. Sci. U.S.A. 83: 2310-2314.

10. Gabizon R, Prusiner SB. (1990) Prion liposomes. Biochem. J. 266: 1-14.

11. Büeler H, Fischer M, Lang Y, et al. (1992) Normal development and behaviour of mice lacking the neuronal cell-surface PrP protein. Nature 356: 577-582.

12. Büeler H, Aguzzi A, Sailer A, et al. (1993) Mice devoid of PrP are resistant to scrapie. Cell 73: 1339-1347.

13. Kascsak RJ, Rubenstein R, Merz PA, et al. (1987) Mouse polyclonal and monoclonal antibody to scrapie-associated fibril proteins. J. Virology 61: 3688-3693.

14. Barry RA, Vincent MT, Kent SBH, Hood LE, Prusiner SB. (1988) Characterization of prion proteins with monospecific antisera to synthetic peptides. J. Immunol. 140: 1188-1193.

15. Gabizon R, McKinley MP, Groth D, Prusiner SB. (1988) Immunoaffinity purification and neutralization of scrapie prion infectivity. Proc. Natl. Acad. Sci. U.S.A. 85: 6617-6621.

16. Serban D, Taraboulos A, DeArmond SJ, Prusiner SB. (1990) Rapid detection of 
Creutzfeldt-Jakob disease and scrapie prion proteins. Neurology 40: 110-117.

17. Prusiner SB, Groth D, Serban A, et al. (1993) Ablation of the prion protein (PrP) gene in mice prevents scrapie and facilitates production of anti-PrP antibodies. Proc. Natl. Acad. Sci. U.S.A. 90: 10608-10612.

18. Krasemann S, Groschup M, Hunsmann G, Bodemer W. (in press) Induction of antibodies against human prion proteins (PrP) by DNA-mediated immunization of $\mathrm{PrP}^{0 / 0}$ mice. J. Immunol. Methods.

19. Krasemann S, Zerr I, Weber T, et al. (1995) Prion disease associated with a novel nine octapeptide repeat-insertion in the PRNPgene. Mol. Brain Res. 34: 173-176.

20. Liljeström P, Garoff H. (1991) A new generation of animal cell expression vectors based on the Semliki Forest Virus replicon. Bio/ Technology 9: 1356-1361.

21. Berglund P, Sjöberg M, Garoff H, Atkins GJ, Sheahan BJ, Liljeström P. (1993) Semliki Forest Virus expression system: Production of conditionally infectious recombinant particles. Bio/Technology 11: 916-920.

22. Davis HL, Michel M-L, Mancini M, Schleef M, Whalen RG. (1994) Direct gene transfer in skeletal muscle: Plasmid DNA-based immunization against the hepatitis B surface antigen. Vaccine 12: 1503-1509.
23. d'Albis A, Couteaux R, Janmot C, Mira J-C. (1989) Myosin isoform transitions in regeneration of fast and slow muscles during postnatal development of the rat. Dev. Biol. 135: 320-325.

24. Kearny JF, Radbruch A, Liesegang B, Rajewski K. (1979) A mouse myeloma cell line that has lost immunglobulin expression but permits the construction of antibody-secreting hybridoma cell lines. J. Immunol. 123: 1548-1558.

25. Peters JH, Baumgarten H. (1990) Monoclonal Antibodies. 2nd Ed. Springer-Verlag, New York, pp. 150-157.

26. Pan K-M, Baldwin $M$, Nguyen $J$, et al. (1993) Conversion of alpha-helices into beta-sheets features in the formation of the scrapie prion proteins. Proc. Natl. Acad. Sci. U.S.A. 90: 10962-10966.

27. Ulmer JB, Donelly JJ, Parker SE, et al. (1993) Heterologous protection against influenza by injection of DNA encoding a viral protein. Science 259: 1745-1749.

28. Xiang ZQ, Spitalnik S, Tran $M$, Wunner WH, Cheng J, Ertl HC. (1994) Vaccination with a plasmid vector carrying the rabies virus glycoprotein gene induces protective immunity against rabies virus. Virology 199: $132-140$. 